\title{
The Role of N-terminal Prohormone of Brain Natriuretic Peptide in the Severity of Coronary Artery Disease (Gensini Score) in Patients with Unstable Angina
}

\section{Seyed Mohammad Hassan Adel ${ }^{1}$, Seyed Mohammadreza Afshani', Nehzat Akiash', Seyed Amir Ali Adel ${ }^{2}$, Mohammad Norouz zadeh $^{\text {1* }}$}

'Department of Cardiology, Atherosclerosis Research Center, Ahvaz Jundishapur University of Medical Sciences, Ahvaz, Iran

${ }^{2}$ Physiology Department, University of Toronto, Toronto, Ontario, Canada

Study Area:Ahvaz, Iran

Coordinates: $31^{\circ} 19^{\prime} 13^{\prime \prime N} ; 48^{\circ} 40^{\prime} 09^{\prime \prime E}$

Keywords: Echocardiography, Ultrasonography, Diagnostic Imaging.

\section{Introduction:}

Coronary Heart Disease (CAD) is the leading cause of death in industrialized and advanced countries and is recognized as the most important factor in preventable mortality in these countries (Haybar \& Zayeri, 2017). Nearly half of all the deaths in industrialized countries and one quarter in developing countries are due to coronary heart disease. By the end of this decade, the mortality rate from heart disease is predicted to surpass even the mortality rate of infectious diseases (Haybar et al., 2018a). In nowadays due to the new diagnostic methods, prevention methods, accurate information about the risk factors of the disease in the world and optimal therapies, the death rate from this disease has decreased, but even in the United States, about one million and five hundred thousand cases Heart disease or fatal heart disease occur annually (WHO, 2002). Considering the advancement of the treatment of this disease and the implementation of aggressive and rescue procedures for heart disease, evaluation of post-treatment morbidity or prediction of the incidence of complications before them is

\section{Abstract}

Coronary artery disease (CAD) is one of the leading causes of mortality in industrialized and advanced countries. This cross-sectional study was aimed to determine the relationship between the severity of CAD (Gensini Score) and Nt-pro BNP in patients with unstable angina on the basis of analytical-descriptive and hospital-based information. The severity of coronary artery stenosis was calculated based on the Gensini Score criteria for coronary artery angiography. In this study, a positive angiographic group had at least one $70 \%$ stenosis in one of the LAD, LCX, RCA vessels, or at least $50 \%$ of the stenosis in the LM. To evaluate Nt-pro BNP, up to 24 hours after Chest pain began, a blood sample was taken from patients, and the amount of Nt-pro BNP was determined by ELISA and by electrochemical luminescence. We found NT-Pro BNP were higher in female in compare to male patients. The mean NT-Pro BNP was significantly higher in angioplasty patients. According to the results of this study, serum NT-Pro BNP level revealed a direct correlation with the severity of coronary artery stenosis in the patients with U.S. and this marker can be used to differentiate patients who need more emergency treatment.

one of the most important research topics in recent years. New criteria that can assess the patients and determine their future conditions with the least invasive procedure are very precise (Mair et al., 1999). Starting stretching on the walls of the heart and increasing the load on the cardiac walls leads to the production of the Pre-Pro-BNP precursor factor, which is broken down to Pro-BNP factor, and after generating the BNP major factor and performing the biological activity as a passive factor, $\mathrm{N}$-Terminal Pro-BNP is excreted in the body. Therefore, the final factor can be used as an indicator for evaluating the main factor. Cardiovascular disease (CVD) has a high degree of mortality and morbidity (Haybar et al., 2018b). There is a strong need for parameters or procedures which provide a comprehensive assessment of the conditions and severity of illness in the short term. The use of NT-Pro BNP to determine the risk and severity of coronary artery disease in patients with unstable angina can be helpful as a quick and cheap test for selecting patients for aggressive interventions. 


\section{Materials and methods:}

This cross-sectional study was conducted on the basis of analytical-descriptive and hospital-based information. Participants in this study were selected from patients referring to the emergency department of Golestan and Imam Khomeini Hospitals in Ahvaz. ST elevation in the patient's heart disease, duration more than 24 hours from onset of symptoms, positive qualitative troponin, patients with CPR, patients with serum creatinine levels of the 2 cases, the company's dissatisfaction with the study, the history of performing $\mathrm{PCI}$ or $\mathrm{CABG}, \mathrm{EF}<50 \%$, NYHA class III \& IV were excluded from the study.

The first 12-lead ECG was prepared, with ECG positive patients presenting with ST depression (1 $\mathrm{mV}$ and above), $\mathrm{T}$ inversion ( $2 \mathrm{mV}$ and more) and $\mathrm{T}$ biphasic and in the absence of ECG negative evidence were considered. Echocardiography was performed by a cardiologist for all patients (echocardiography device used by Philips EPIQ7).

Evaluation of coronary artery stenosis in angiography: the severity of coronary artery stenosis was calculated on the basis of the Gensini Score criteria and according to the angiography performed by a cardiologist during admission (angiography device used by Siemens ARTIS ZEE with Pure 2017). Scoring is based on the severity of the stenosis and the functional importance of the stenosis in terms of hemorrhage to the myocardium. The severity of stenosis is classif ied as 1, 2, 4, 8, 16, 32 for $25 \%, 50 \%, 75 \%, 90 \%, 99 \%$, $100 \%$, respectively, in the diameter of the lumen of the vein. The functional significance of the site of stenosis is also considered as follows: left main coronary artery $\times 5$ Proximal segment of the left anterior descending coronary artery $(L A D) \times 2.5$. proximal segment of the left circumflex artery $($ LCX $) \times 2.5$. mid segment of the LAD $\times 1.5$. right coronary artery (proximal, mid, distal), posterior left ventricular (PLV), posterior descending artery, distal segment of the LAD, first diagonal, distal segment of the LCX and obtuse marginal artery $\times 1$. second diagonal $\times 0.5$. The final score is obtained as a sum of the product of the severity of the tightness in the importance of the site of the stench (Fig.-1). In this study, the positive angiography group had at least one stenosis about $70 \%$ in one of the LAD, LCX, RCA vessels, or at least $50 \%$ of stenosis in the LM. Also, if no coronary artery disease was detected in more than $50 \%$ of patients, no coronary artery stenosis, stenosis of at least $50 \%$ of patients with $1 \mathrm{VD}, 2$ narrow stenosis of at least $50 \%$ in 2 vascular patients $2 \mathrm{VD}$ and 3 Stenosis was considered to be at least $50 \%$ in 3 vesseles.

Measurement of serum Nt pro-BNP: for evaluation of $\mathrm{Nt}$ pro BNP, a blood sample was taken from patients for up to 24 hours after chest pain began, and after centrifugation, the obtained plasma was freeze at $20^{\circ} \mathrm{C}$. The samples were melted, and the amount of Nt pro-BNP was determined by ELISA method and Luminescence Electrochemical Device.

Data analysis: in this study, descriptive statistics including mean, standard deviation, frequency, and frequency were reported. Pearson correlation coefficient was used to determine the correlation between the true parameters and the prevalence of it. In the case of abnormal data, Spearman's correlation coefficient was used. Data analysis was performed using SPSS version 22 software and the significance level was $<0.05$.

\section{Results:}

In this study 160 patients were examined; $58.7 \%$ of the patients were male and $41.3 \%$ were female. Further, $51.7 \%$ of the patients had positive coronary angiography results. The mean age of the patients was $54.4 \pm 10.3$ years (at least 33 and at most 77 years). The mean age of patients with coronary angiography was slightly higher ( 57 years Vs. 52 years). But this difference was not statistically significant $(\mathrm{p}>0.215)$. In this study, 71 patients (44.3\%) had positive ECG. In the angioplasty group, the ECG was slightly more than the angioplasty group ( $45.7 \% \mathrm{Vs} 42.7 \%$ ), but this difference was not statistically significant. The average Gensini score for patients was $31.2 \pm 28.6$ (with a minimum of $\mathrm{o}$ and a maximum of 119). The mean Gensini score in the coronary angiography group was signif icantly higher than those with angiography (50.7 Vs 13) and this difference was statistically significant $(\mathrm{p}<\mathrm{o.0oo})$. Further, serum NT-Pro BNP concentration was higher in female patients as compared to male patients (346 n/L Vs. $234 \mathrm{n} / \mathrm{L}$ ), but this difference was not statistically significant ( $>>0.192)$. There was no significant relationship between age and NT-Pro BNP concentration ( $p>0.194$ ). The mean NT-Pro BNP concentration in this study was $299 \pm 241.6 \mathrm{n} / \mathrm{L}$ (with a minimum of $104 \mathrm{n} / \mathrm{L}$ and a maximum of $1000 \mathrm{n} / \mathrm{L}$ ). The mean serum NT-Pro BNP concentration was higher in patients with angioplasty $(578 \mathrm{n} / \mathrm{L}$ Vs $210 \mathrm{n} / \mathrm{L})$ and this difference was statistically significant $(\mathrm{p}<0.000)$. The serum NT-Pro BNP concentrations in patients with $3 \mathrm{VD}$, 2VD, 1VD and without stenosis were 836 n / L, $769 \mathrm{n} / \mathrm{L}, 457$ $\mathrm{n} / \mathrm{L}$, and $210 \mathrm{n} / \mathrm{L}$ respectively. This difference is statistically significant $(\mathrm{p}<\mathrm{o} .001)$. Considering that in this study NTPro BNP has a statistically significant relationship with positive angiography of patients $(\mathrm{p}<0.000)$. We can use the ROC chart, sensitivity, specif icity and best cutting point for NT-Pro BNP would be used to predict the positive angiography and eventually requires patients to take more emergency measures. Figure-2 is the ROC chart and the Coordinates table for the data in this study. Given the AUC calculated for this data, 0.932, NT-Pro BNP has the potential to predict angiography positivity in patients with high potency. Further, NT-Pro BNP serum levels at 179 n/L with a sensitivity of $73.3 \%$ and a $50 \%$ specif icity were able to diagnose the angiography of patients with UA. There was a significant correlation between Gensini score and NT-Pro BNP concentration $(\mathrm{p}<0.000)$. The calculated Pearson coefficient was 0.5 , indicating a direct and intermediate relationship between these two variables as increased 
serum NT-Pro BNP with increasing Gensini score.

Table-1: Study variables for the results of coronary angiography

\begin{tabular}{|c|c|c|c|c|}
\hline \multirow{2}{*}{\multicolumn{2}{|c|}{ Variables }} & \multicolumn{2}{|c|}{ Angiographic results } & \multirow[t]{2}{*}{ P-value } \\
\hline & & Posetive & Negative & \\
\hline \multirow{2}{*}{\multicolumn{2}{|c|}{$\begin{array}{l}\text { Age } \\
\text { ECG }\end{array}$}} & 57 & 52 & $>0.215$ \\
\hline & & & & \\
\hline \multirow[t]{2}{*}{ (n) } & Posetive & 38 & 33 & $>0.566$ \\
\hline & Negative & 45 & 44 & \\
\hline \multicolumn{2}{|c|}{ Gensini (Mean) } & 50.7 & 13 & $<0.000$ \\
\hline \multicolumn{2}{|c|}{ NT-Pro BNP (Mean)n/L } & 578 & 210 & $<0.000$ \\
\hline
\end{tabular}

Table-2: Process to calculate Gensini score

\begin{tabular}{llll}
\hline LM $: 5$ & p-LAD $: 205$ & $25 \%: 1$ & $50 \%: 2$ \\
m-LAD $: 1.5$ & d-LAD $: 1$ & $75 \%: 4$ & $90 \%: 8$ \\
D1: 1 & D2 $: 0.5$ & $99 \%: 15$ & $100 \%: 32$ \\
p-LCX $: 2.5$ & d-LCX $: 1$ & Gensisi score= sum of \\
OM $: 1$ & p-RCA $: 1$ & (Functional score X Stenosis score) \\
m-RCA $: 1$ & d-RCA : 1 & \\
PDA $: 1$ & PLV : 1 & \\
\hline
\end{tabular}

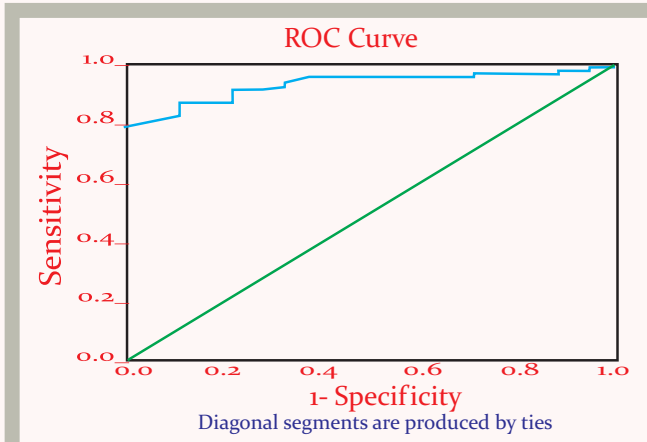

Figure-1: ROC Diagram to evaluate the sensitivity and specificity of NT-Pro BNP in predicting positive angiography of patients.

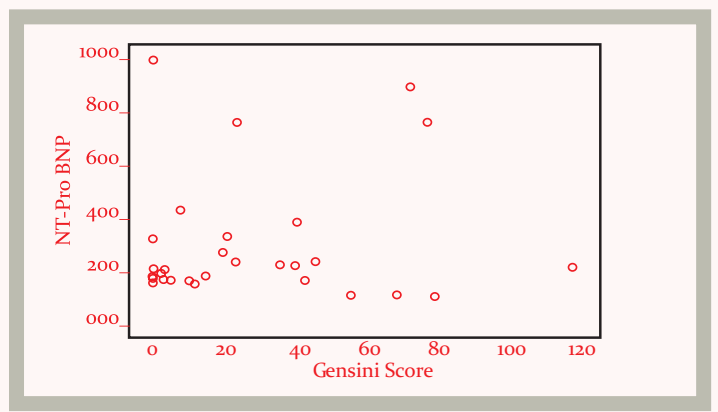

Figure-2: : Gensini Score Spot and Serum Concentration of NT Pro BNP

\section{Discussion:}

In our study, we found a signif icant correlation between the Gensini score and NT-Pro BNP concentration and Pearson's calculated coefficient was 0.5 ( $p>0.000)$ which indicates a direct and intermediate relationship between these two variables. With the increasing Gensini score, the serum concentration of NT-Pro BNP also increased. The results of this study correlate with the results of previous studies on the relationship between Gensini score and serum NT-Pro BNP levels Wei et al. (2012), the serum NT-Pro BNP concentration also increases with an increase in ischemic heart rate. Further, Rageb et al. (2017) reported that NT-Pro BNP serum level was higher in patients with coronary artery angiography with $50 \%$ and higher concentrations of NTPro BNP with increasing severity of coronary arteries damage. Wu et al. (2014) reported there was a significant direct correlation between the Gensini score and serum NTPro BNP concentration. Based on the results of the present study and the results of previous studies on the increase of the severity of coronary artery stenosis, and as a result of the increase in Gensini score, NT-Pro BNP secret from the heart cells and NT-Pro BNP could be referred to use as a marker to determine the severity of the conflict in CAD. The results of this study and the previous study are consistent with the fact that the NT-Pro BNP serum level does not correlate with the gender of the patient and increases the concentration of the patient's serum independently of the patient's gender by other causes, such as the severity of coronary artery bypass grafting (Wu et al., 2014). Kragelund et al. (2005) suggested that there was a significant correlation between serum NT-Pro BNP concentration and the number of vessels involved $(\mathrm{p}<0.001)$. They also showed a signif icant relationship between NT-Pro BNP and the severity of coronary artery disease in angiography. Shahabi et al. (2011) showed there was a strong correlation between serum NT-Pro BNP levels and the severity of coronary involvement. Our results and the results of previous studies indicate that increased NT-Pro BNP concentration is indicative of high-intensity coronary artery stenosis and increased serum NT-Pro BNP levels in patients with more entrapped veins, and this marker Is able to assist doctors in predicting more and more severe vessel involvement in a patient referring to the hospital (Kragelund et al., 2005). Considering that in our study NTPro BNP was significantly correlated with positive angiography of patients $(\mathrm{p}<\mathrm{0.00o})$, and considering the AUC 0.932 for the diagnostic power of this marker, NT-Pro BNP with high power The ability to predict the angiography is positive in patients. Further, NT-Pro BNP can detect positive angiography in patients with UA (Shahabi et al., 2011). Yehia et al. (2012) suggested that the calculated area below the graph was 0.71 and the NT-Pro BNP with a cutout point of $100 \mathrm{n} / \mathrm{L}$ had a sensitivity of $65 \%$ and a specif icity of $72 \%$, and at the cut point of $\mathrm{n} / \mathrm{L} 120$, the sensitivity of the test was $54 \%$ and the specificity was $74 \%$. (Özmen et al. (2012) showed that the area under the graph was 0.776 and at the $116.5 \mathrm{n} / \mathrm{L}$ cut point, the sensitivity and specificity of this marker were able to detect the presence of coronary artery occlusion was reported to be $90 \%$ and $55.7 \%$, respectively. Several studies showed the area under the graph was 0.56 . Considering the results of this study and the results of previous studies showed the NT-Pro BNP serum marker with a specific sensitivity and specificity is able to differentiate patients with more risky conditions and help 


\section{ORIGINAL ARTICLE}

doctors to take more aggressive measures.

Conclusively, NT-Pro BNP serum levels have a direct correlation with the severity of coronary artery stenosis in patients with US and this marker can be used to differentiate the patients who need more emergency treatment. The limitations of the study were the small size of the population studied to determine a curable method for the general population to detect unstable angina with higher risk. The lack of uniformity of patient sampling time in the onset of pain and the timing of the failure to determine the maximum concentration of NT-Pro BNP in the serum, which certainly affects the results. Due to the prolonged half-life of the NT-Pro BNP plasma, this problem was partly overlooked. The less accurate Gensini score was to detect stenoses compared to IVUS and FFR methods, which, given the prevalence in recent years, would provide more accurate results.

\section{References:}

Haybar, H. \& Zayeri, Z.D. (2017): The value of using polymorphisms in anti-platelet therapy. Front. Biol., 12(5):349-356.

Haybar, H., Jalali, M.T. \& Zayeri, Z.D. (2018a): What Genetics Tells us about Cardiovascular Disease in Diabetic Patients? Cardiovasc. Haematol. Disord. Drug Targets. 18(2):147152.

Haybar, H., Parsa, S.A., Khaheshi, I. \& Zayeri, Z.D. (2018b): Pentraxin level is the key to determine primary Percutaneous Coronary Intervention (PCI) or fibrinolysis. Cardiovasc. Hematol. Disord. Drug Targets, 19(2):160-168.

Kragelund, C., Grønning, B., Køber, L., Hildebrandt, P. \& Steffensen, R. (2005): N-terminal proB-type natriuretic peptide and long-term mortality in stable coronary heart
Ambient Science, 2020: Vol. 07h(1); 10-13 DOI:10.21276/ambi.2020.07h.1.oa02

disease. N. Engl.J.Med., 352(7):666-675.

Mair, J., Friedl, W., Thomas, S. \& Puschendorf, B. (1999): Natriuretic peptides in assessment of left-ventricular dysfunction. Scand. J. Clin. Lab. Invest, 59(sp.230):132142 .

Özmen, B., Özmen, D., Parildar, Z., Mutaf, I. \& Bayindir, O. (2007): Serum N-terminal-pro-B-type natriuretic peptide (NT-proBNP) levels in hyperthyroidism and hypothyroidism. Endocr. Res., 32(1-2):1-8.

Rageb, F., Rageb, D. \& Moafy, A. (2017): Detection of severity of acute coronary syndrome using $\mathrm{N}$ terminal PRO-BNP and as a prognostic marker. J. Am. Sci., 13:43-48.

Shahabi, V., Moazenzadeh, M., Azimzadeh, B.S., Nasri, H., Afshar, R.M., Shahesmaili, A. \& Rashidinejad, H. (2011): Relationship between serum N-terminal Pro Brain Natriuretic Peptide (NT-Pro BNP) level and the severity of coronary artery involvements. L. Res. Med. Sci., 16(2):143-148.

Wei, G., Ningfu, W., Xianhua, Y., Liang, Z., Jianmin, Y., Guoxin, T. \& Peng, X. (2012): N-terminal pro-B-type natriuretic peptide is associated with severity of the coronary lesions in unstable angina patients with preserved left ventricular function. $J$. Interven. Cardiol., 25(2):126-231.

WHO (2002): The world health report 2002: reducing risks, promoting healthy life. Pub. by: World Health Organization.

Wu, N., Ma, F., Guo, Y., Li, X., Liu, J., Qing, P., Xu, R., Zhu, C., Jia, Y., Liu, G., Dong, Q., Jiang, L. \& Li, J. (2014): Association of Nterminal pro-brain natriuretic peptide with the severity of coronary artery disease in patients with normal left ventricular ejection fraction. Chin. Med. I., 127(4):627632.

Yehia, A., Rizk, A.A., Nouh, S. \& Moutawea, A. (2012): Relationship between NT Pro BNP blood level and coronary artery disease in patients having chronic stable angina and normal lv diastolic function. $\underline{A A M I}$. 10(3):197-209. 